\title{
Studying Individual Aging in an Interindividual Context: Typical Paths of Age-Related, Dementia-Related, and Mortality-Related Cognitive Development in Old Age
}

\author{
Martin Lövdén \\ Max Planck Institute for Human Development \\ Rolf Adolfsson \\ Umeå University
}

\author{
Lars Bergman \\ Stockholm University
}

\author{
Ulman Lindenberger \\ Max Planck Institute for Human Development
}

\author{
Lars-Göran Nilsson \\ Stockholm University
}

\begin{abstract}
This study has 2 objectives: (a) to explore typical paths of cognitive development associated with aging, terminal decline, and dementia and (b) to promote and illustrate an individual-oriented approach to the study of cognitive aging based on longitudinal panel data from a population-based sample $(N=500$; age range $_{\mathrm{T} 1}=60-80$, where $T$ refers to time) tested at 3 occasions 5 years apart. Results document interindividual differences in multivariate patterns of change. Although cognitive changes generally covary, the present study indicates that subgroups of individuals develop along different paths characterized by selective changes in subsets of cognitive functions. Typical progression of dementia followed a developmental cascade from low declarative memory, via low functioning across all observed cognitive measures, to dementia diagnosis, and finally, death.
\end{abstract}

Keywords: episodic memory, semantic memory, dementia, terminal decline, pattern-oriented analysis

This study had two objectives: (a) to explore typical paths of cognitive development associated with aging, terminal decline, and dementia in old age and (b) to encourage an individualoriented approach to the study of cognitive aging. Because this approach is relatively nonstandard in cognitive aging research, it might be appropriate to outline some of its features.

An individual-oriented approach to the study of development is not new; on the contrary, key characteristics of this approach have been delineated by proponents of a person-oriented perspective

Martin Lövdén and Ulman Lindenberger, Center for Lifespan Psychology, Max Planck Institute for Human Development, Berlin, Germany; Lars Bergman and Lars-Göran Nilsson, Department of Psychology, Stockholm University, Stockholm, Sweden; Rolf Adolfsson, Department of Clinical Sciences, Division of Psychiatry, Umeå University, Umeå, Sweden.

The Betula study is funded by the Bank of Sweden Tercentenary Foundation Grants 1988-0082:17 and J2001-0682; Swedish Council for Planning and Coordination of Research Grants D1988-0092, D1989-0115, D1990-0074, D1991-0258, D1992-0143, D1997-0756, D1997-1841, D1999-0739, and B1999-474; Swedish Council for Research in the Humanities and Social Sciences Grant F377/1988-2000; Swedish Council for Social Research Grants 1988-1990:88-0082 and 311/1991-2000; and Swedish Research Council Grants 2001-6654, 2002-3794 and 2003-3883.

Correspondence concerning this article should be addressed to Martin Lövdén, Center for Lifespan Psychology, Max Planck Institute for Human Development, Lentzeallée 94, D-14195, Berlin, Germany. E-mail: loevden@mpib-berlin.mpg.de (e.g., Allport, 1937; Bergman, Magnusson, \& El-Khouri, 2003; Block, 1971; Magnusson, 2001), life span developmental theoreticians and methodologists (e.g., Baltes \& Nesselroade, 1979; Baltes, Reese, \& Nesselroade, 1977; see also Hertzog, 1985; Lövdén \& Lindenberger, 2005; Molenaar, Huizenga, \& Nesselroade, 2003), and developmental systems theoreticians (e.g., Ford \& Lerner, 1992; Gottlieb, 1992; Wohlwill, 1973; see also S.-C. Li, 2003). Broadly viewed, four related perspectives guide the individual-oriented agenda.

First, understanding development requires a multivariate and interactionist perspective, viewing individuals, or subsystems at different levels, as indivisibles. Rather than standing for themselves, the significance of different parts in a system like cognition is held to emerge from interactions among the parts and from the role the parts play in the total functioning of the system. In other words, an individual's level of performance on a particular ability needs to be interpreted in the context of this individual's ability profile. The intraindividual performance profile is, from this perspective, the essential indivisible unit of analysis (e.g., Bergman et al., 2003; Magnusson, 2001). For example, low episodic memory ability may have different developmental meanings for individuals with high or low semantic memory.

Second, individuals can be described as open systems, continuously variable, and achieving more or less stable equilibria through, among other principles, self-organization. In other words, individual development is viewed as a dynamic process in which 
certain states (value patterns) function as attractors and tend to occur frequently in a sample of individuals (e.g., Bergman \& Magnusson, 1997). Furthermore, a system's state at time $t$ is held to be dependent, linearly or nonlinearly, on its state at time $t-1$. In other words, change from $t-1$ to $t$ is dependent on the prior state of the system (e.g., Smith \& Thelen, 2003).

The third point highlights that standard measurement models, such as standard cross-sectional and longitudinal factor analyses, are concerned with interindividual differences. Therefore, these models tell us little about how variables are related within individuals (e.g., Borsboom, Mellenbergh, \& van Heerden, 2003; Jones \& Nesselroade, 1990; Molenaar et al., 2003). For example, a strong association between sensory functioning and processing speed, as revealed by an analysis of interindividual differences (e.g., Baltes \& Lindenberger, 1997), does not prescribe how these variables are coupled within individuals (cf. K. Z. H. Li \& Lindenberger, 2002).

The fourth perspective questions an implicit acceptance of a "uniformity-of-nature assumption" (Borsboom et al., 2003, p. 215). That is, standard multivariate statistics and much of the accompanying theory assume that structural relations among variables are homogeneous over individuals. Several lines of evidence concerning cognitive change in old age illustrate the problems with an overly strong reliance on this assumption. For example, Sliwinski, Hofer, and Hall (2003) showed that correlations among agebased cognitive changes were higher in a group of individuals with preclinical dementia, as compared with a group of nondemented individuals. The moderate correlations among changes observed in the mixed sample were partly attributable to the aggregation of these two subgroups. Further sources of heterogeneity of development in older samples include age-related change versus mortalityassociated change (e.g., Bosworth \& Schaie, 1999; Kleemeier, 1962; Riegel \& Riegel, 1972; Singer, Verhaeghen, Ghisletta, Lindenberger, \& Baltes, 2003; Small, Fratiglioni, von Strauss, \& Bäckman, 2003). To illustrate, Singer et al. (2003) derived crosssectional age gradients in processing speed and knowledge at baseline from a sample ( $n=516$; age range $\left.{ }_{\mathrm{T} 1}=70-103\right)$ that was followed up on after 4 and 6 years. Age gradients for two different subsamples were compared: gradients for the total sample and for a sample of individuals who survived and participated in all follow-up waves $(n=132)$. The former sample included participants in closer proximity to death and more participants suffering from health disorders, whereas the latter consisted of select and relatively healthy individuals. Negative age gradients were observed for processing speed in both subsamples, but knowledge did only decline significantly in the total sample. Thus, decline in processing speed accompanied by stable knowledge might be a normatively age-related multivariate developmental path, whereas decline in both abilities might be associated with impending death (see also Small et al., 2003). Of course, if concerted declines in speed and knowledge were primarily mortality related, one would expect most individuals to sooner or later enter this developmental path. Hence, as widely recognized, an age-based analysis may conceal developmental patterns that are common across individuals (e.g., Wohlwill, 1970). Specifically, the central question in cognitive aging research- "Does it all [cognitive functions] go together when it goes?" (Rabbitt, 1993, p. 385)—may have different answers for different people: It might go together for some, or even for most individuals, but not necessarily for all, and, for different individuals, it might all go together at different chronological ages. Such heterogeneity in developmental trajectories will go unnoticed as long as researchers tacitly rely on methods that endorse rather than test the uniformity-of-nature assumption.

The perspectives guiding the individual-oriented approach are not about statistical issues per se-they are about adopting a particular perspective for the study of development. Statistical issues come into play when choosing a measurement model or an empirical design that matches the perspective. From what has been said above, such an individual-oriented methodological approach should preferably capture the dynamic, multivariate, and interactionist nature of individual development; clearly separate intraindividual and interindividual differences; and explicitly structure heterogeneity.

In light of the above considerations, it can be argued that understanding development demands the study of each individual's multivariate intraindividual change, followed by careful generalization based on a combination of several such studies. Empirically, such an approach means collecting many variables, within several individuals, many times. Couched within the context of studying short-term changes in behavior, this multivariate, single-subject, repeated measures design has been advocated by, for example, Nesselroade (e.g., Jones \& Nesselroade, 1990; Nesselroade \& Ford, 1985) and Molenaar (e.g., Molenaar et al., 2003), but the design is also at the core of studying long-term individual development (e.g., Nesselroade \& Ghisletta, 2000; Valsiner, 1984). For example, intensive short-term longitudinal information might be necessary for separating between short-term fluctuations in behavior and more enduring changes (Nesselroade \& Salthouse, 2004). However, researchers are rarely fortunate enough to possess such intensive longitudinal information. Furthermore, interest in interindividual differences and the existence of a great number of ongoing longitudinal studies featuring many older individuals, many variables, but only a few assessments that are widely separated in time motivates an attempt to come closer to the desirable measurement model with other methods.

Pattern-oriented methods offer one possible avenue. Many such methods aim at classifying individuals into homogeneous groups on the basis of similarities among individuals' profiles of values on the variables under study. Examples include longitudinal configural frequency analysis (von Eye, 1990), methods based on cluster analyses (e.g., Bergman et al., 2003; see Schaie, 1989, for an example of cognitive aging research), latent profile analysis, latent class analysis, and the longitudinal extension, latent transition analysis (e.g., Collins \& Wugalter, 1992). All of these methods attempt to grasp the extent and nature of sample heterogeneity in multivariate patterns of change. Furthermore, whereas many standard multivariate approaches may represent heterogeneity with multigroup models or by including interaction terms, the patternbased approach offers an alternative for structuring unknown heterogeneity and interactions.

Recent developments in the multilevel modeling and latent growth curve modeling frameworks offer other methodological avenues (see Collins \& Sayer, 2001; Little, Schnabel, \& Baumert, 2000; Muthén \& Muthén, 2000, for overviews). These approaches combine the classic growth curve modeling approach with the pattern-oriented approach; to not only represent, but also structure 
interindividual differences in change (e.g., Muthén \& Muthén, 2000; see also Nagin \& Tremblay, 2001). For example, one group may have a linear growth curve, whereas another group may follow a quadratic curve.

The methodological approaches summarized above all have their advantages, limitations, and assumptions. In this study, we opt for a pattern-oriented approach based on cluster analysis. Bergman (1998; Bergman et al., 2003) developed such a procedure for the analysis of long-term longitudinal panel data: linking of clusters after removal of a residue (LICUR). To the procedure's advantages belong robustness, flexibility, suitability for long-term longitudinal panel data, and the substantive questions posed in this study. Moreover, in contrast to the often univariate nature of the recent developments in the growth curve modeling framework, it is inherently a multivariate approach. Briefly, the procedure includes an attempt, at each measurement occasion (or age), to form clusters (i.e., groups) of individuals who are homogenous (with respect to the value pattern on the included variables) within a cluster but heterogeneous across clusters. Then, the different clusters are matched across time (e.g., the time $t$ clusters are matched to the $t+1$ clusters) and typical individual transitions across clusters are studied over time. Though results are summarized at a group level, the procedure is person oriented because individuals belonging to the same cluster at a given measurement occasion have approximately the same intraindividual pattern of values. The intraindividual pattern for an individual at one assessment is then related to the intraindividual pattern at a later assessment.

Substantively, the first major research question addressed in this article concerns the debate about the degree to which age-related changes in different cognitive functions are independent of each other. Support for a relatively unitary account of cognitive aging has primarily come from cross-sectional studies of ageheterogeneous samples showing that age differences in many cognitive abilities are well predicted by the shared variance among these abilities and by interindividual differences in biomarkers such as sensory functioning (e.g., Anstey \& Smith, 1999; Lindenberger \& Baltes, 1994; Luszcz \& Bryan, 1999; Salthouse \& Czaja, 2000; Verhaeghen \& Salthouse, 1997; but see Allen et al., 2001; Hofer, Berg, \& Era, 2003; Hofer \& Sliwinski, 2001; Lindenberger \& Pötter, 1998; Schmiedek \& Li, 2004). Similarly, results from several longitudinal studies suggest that, although interindividual differences in change are relatively limited in select samples relatively free from pathology, correlations among changes in different cognitive abilities tend to be high (e.g., Hultsch, Hertzog, Dixon, \& Small, 1998; Lövdén, Rönnlund, et al., 2004; Wilson et al., 2002).

As previously noted, the debate regarding the prominence of a general factor in cognitive aging has often been implicitly framed in the context of a strong uniformity-of-nature assumption, in the sense that structural associations across cognitive variables have been assumed to be invariant across individuals (but see Singer et al., 2003; Sliwinski, Hofer, \& Hall, 2003). To provide a different perspective on this issue, we explored intraindividual multivariate patterns of change with the expectation of finding typical patterns consistent with concerted cognitive change for some individuals but, for other individuals, also patterns consistent with selective change in a subset of the included measures.
A second main empirical question concerned a major source of interindividual and intraindividual differences in cognitive development in old age: dementia. Cognitive impairments several years prior to diagnosis have been shown to be sensitive markers of later diagnosis (i.e., preclinical deficits; e.g., Bäckman, Laukka, Wahlin, Small, \& Fratiglioni, 2002; Bäckman, Small, \& Fratiglioni, 2001; Elias et al., 2000; Fabrigoule et al., 1998; Jacobs et al., 1995; Small, Herlitz, Fratiglioni, Almqvist, \& Bäckman, 1997; see Spaan, Raaijmakers, \& Jonker, 2003, for a review focused on memory). Preclinical cognitive deficits tend to be rather global (e.g., Bäckman et al., 2002; Fabrigoule et al., 1998), but impairments in episodic memory are perhaps the most prominent finding (e.g., Bäckman et al., 2001; Small et al., 1997). Furthermore, it has been suggested that impairments in functions that are normally well preserved in old age, such as semantic memory, may distinguish dementia from aging (e.g., Branconnier, Cole, Spera, \& DeVitt, 1982; Hodges \& Patterson, 1995; Small et al., 1997; see also Spaan et al., 2003). A main empirical objective of this study was to explore typical multivariate cognitive states preceding dementia diagnosis, with the expectation of finding such states to be characterized by global deficits. Furthermore, we aimed at exploring typical 10-year developmental paths toward dementia diagnosis. On the basis of the expected proximal preclinical pattern of global cognitive deficits, we expected that developmental paths from a still relatively differentiated cognitive system, but possibly low declarative memory functioning (i.e., episodic and semantic memory), toward low functional levels across the system would be typical for the long-term preclinical progression of dementia.

Finally, terminal decline, denoting an association between proximity to death and cognitive performance (Kleemeier, 1962; Riegel \& Riegel, 1972), constitutes another important source of interindividual and intraindividual differences in old age. Several studies suggest that cognitive changes associated with terminal decline may reflect other causal structures than age-related changes; for example, specific cognition-influencing diseases and global breakdowns of the biological system (see Berg, 1996; Small \& Bäckman, 1999, for overviews). As noted above, several recent studies have also suggested that the multivariate cognitive pattern of change in the terminal decline phase may differ from age-related changes. Specifically, the terminal decline phase may be characterized by a pattern of global cognitive change, including decline in crystallized abilities such as semantic memory, which may be relatively unaffected by aging-related processes (e.g., Singer et al., 2003; Small et al., 2003; see also Lövdén \& Lindenberger, 2005). Therefore, a final empirical objective was to provide further knowledge concerning the multivariate patterns of change in terminal decline.

The data considered in this study emanate from the first, second, and third measurement occasions, each separated by 5 years, of the Betula study (Nilsson et al., 1997, 2004). The sample was population based and included 500 individuals at baseline (age range $=$ 60-80). All individuals were initially free from dementia diagnosis. We included cognitive measures that were selected to represent major building blocks and products of the cognitive system: broad fluid abilities, episodic memory, and broad crystallized abilities (or, semantic memory). 


\section{Method}

For detailed information concerning the overall design of the Betula study, participant characteristics of the original samples, and the specific test procedures, see Nilsson et al. (1997) and Nilsson et al. (2004). Only methodological features pertinent to this study are described below.

\section{Participants}

The sample was restricted to five cohorts: $60,65,70,75$, and 80 years old at time of first assessment (T1). At T1, this sample consisted of 500 individuals, 100 in each cohort. All participants were recruited by random sampling from the population registry in Umeå, a city with about 100,000 inhabitants in Northern Sweden. Individuals were excluded if they (a) had a dementia diagnosis, (b) suffered from mental retardation, (c) suffered from severe sensory handicaps, or (d) had another language than Swedish as their native tongue.

Nilsson et al. (1997) compared participants in the Betula study ( $n=$ 1,000 in the total T1 sample) and nonparticipants (contacted individuals unwilling to participate; $n=481$ ) on a number of background variables. The results showed no differences between participants and nonparticipants on most of the variables included, but participants were employed to a greater extent and had higher incomes. As compared with the general population in Sweden, participants had higher levels of education and higher incomes, which is expected from the fact that a university is located in Umeå. Thus, the general impression was that the sample is highly representative of the target population.

Five years later, at the second assessment (T2), the effective sample was reduced to 387 participants. That is, 113 individuals (22.6\%) were unwilling or unable to return for the complete $\mathrm{T} 2$ assessment. Ten years later, at the third assessment wave (T3), 225 participants remained in the effective sample. Summary characteristics of the sample as a function of measurement occasion are displayed in Table 1. Note that the means for the cognitive variables are relatively stable over measurement occasions, which is attributable to longitudinal selectivity effects. Because the main analyses focus on these effects, we performed no standard selectivity analysis.

We explored three different reasons for not being included in the effective sample at each assessment wave: death, dementia diagnosis, and experimental dropout (i.e., refusal, moved, ill, not available, or missing values on all relevant measures). Individuals in a dead category died at some point prior to a follow-up assessment (i.e., T2 or T3). Naturally, this category absorbed individuals who at a prior occasion (i.e., T2) belonged to the other two categories (i.e., dropout and dementia diagnosis). Individuals in the dementia group received a dementia diagnosis in connection or prior to a specific measurement occasion. The individuals with dementia diagnosis were difficult to test with the Betula cognitive battery, which resulted in unacceptable amounts of missing values on the variables included in this study. Therefore, this category absorbed individuals from the effective sample. Furthermore, to the extent it was possible to establish whether a dementia diagnosis had been issued external to the study, the category absorbed individuals from the group of individuals dropping out between any two occasions for experimental reasons. Because it has lately been suggested that vascular dementia and Alzheimer's disease share many features (e.g., Kalaria \& Ballard, 1999) — the cognitive impairments are, for example, strikingly similar (e.g., Almqvist, Fratiglioni, Agüero-Torres, Viitanen, \& Bäckman, 1999) — we chose to treat the dementia cases as a single category (cf. Bäckman et al., 2002; Fabrigoule et al., 1998). Individuals classified as experimental dropouts were still alive at a specific occasion and, to the extent it was possible to ascertain, nondemented-but they were unwilling or unable to participate for some other reason.

Out of the 113 individuals not included in the effective sample at T2, 48 were dead, 27 had a dementia diagnosis, and 38 belonged to the experimental dropout category. Out of the 275 individuals not tested at T3, 73 belonged to the experimental dropout category, 149 were dead, and 53 had a dementia diagnosis. Summary characteristics of the dropout groups are included in the Results section (see Table 4, which will be discussed later in the text).

A dementia diagnosis was established by the following procedure. First, screening criteria narrowed down the number of participants to be considered for extensive diagnosis evaluation. Specifically, those fulfilling one or more of the following criteria were referred to a specialist in neuropsychiatry: (a) suspected dementia signs observed by the nurse or the psychologist serving as experimenters, (b) a Mini-Mental State Exam score (Folstein, Folstein, \& McHugh, 1975) below 24, and (c) a decline of three or more Mini-Mental State Exam points over the 5-year follow-up intervals. On the basis of further extensive examination and the Diagnostic and Statistical Manual of Mental Disorders (4th ed., text rev.; American Psychiatric Association, 2000) criterion, a neuropsychiatrist made the decision whether a clinical diagnosis of dementia was warranted. Among those who refused or were unable to participate in an assessment wave, medical records, available from various clinics including psychiatry, neurology, and geriatrics, were examined to establish whether a dementia diagnosis had been issued external to the study.

\section{Procedure and Measures}

At all three assessment waves, the measures were collected during two test sessions, both which lasted for about 1.5 to $2 \mathrm{hr}$ for each participant The first session consisted mainly of health examination and questionnaires. The second session comprised an extensive cognitive measurement battery. All participants were tested individually and were requested to use hearing aids or glasses if used. We selected Block Design (Wechsler,

Table 1

Summary Characteristics for the Effective Sample at Each Measurement Occasion

\begin{tabular}{|c|c|c|c|c|c|c|}
\hline \multirow[b]{2}{*}{ Variable } & \multicolumn{2}{|c|}{$\mathrm{T} 1(N=500)^{\mathrm{a}}$} & \multicolumn{2}{|c|}{$\mathrm{T} 2(N=387)^{\mathrm{b}}$} & \multicolumn{2}{|c|}{$\mathrm{T} 3(N=225)^{\mathrm{c}}$} \\
\hline & $M$ & $S D$ & $M$ & $S D$ & $M$ & $S D$ \\
\hline Age & 70.0 & 7.1 & 74.1 & 7.0 & 76.7 & 6.4 \\
\hline Years of education & 8.0 & 3.1 & 8.1 & 3.2 & 8.4 & 3.2 \\
\hline MMSE & 27.3 & 2.1 & 26.9 & 2.4 & 27.3 & 1.9 \\
\hline Block Design & 50.0 & 10.0 & 49.0 & 10.2 & 48.4 & 9.7 \\
\hline Episodic memory & 50.0 & 10.0 & 48.8 & 11.2 & 50.6 & 10.6 \\
\hline Semantic memory & 50.0 & 10.0 & 50.5 & 10.4 & 51.1 & 9.5 \\
\hline
\end{tabular}

Note. $\mathrm{T}=$ time; MMSE $=$ Mini Mental State Examination (Folstein, Folstein, \& McHugh, 1975).

${ }^{\mathrm{a}} n_{\text {men }}=240$ and $n_{\text {women }}=260 .{ }^{\mathrm{b}} n_{\text {men }}=182$ and $n_{\text {women }}=205 .{ }^{\mathrm{c}} n_{\text {men }}=102$ and $n_{\text {women }}=123$. 
1981), four measures of episodic memory, and four indicators of semantic memory as measures of cognitive performance. The individual indicators of semantic and episodic memory were selected because of their satisfactory psychometric properties and longitudinal availability (cf. Lövdén, Rönnlund, et al., 2004; Rönnlund, Nyberg, Bäckman, \& Nilsson, 2005).

Block Design. The obtained raw scores from the Block Design test from the Wechsler Adult Intelligence Scale-Revised (Wechsler, 1981) were used. In this task, participants are, under speeded conditions, required to place red and white blocks such that they form the same pattern shown on a target paper.

Episodic memory functioning. Three measures of recall performance and one measure of recognition performance were used as indicators of episodic memory functioning (cf. Nyberg et al., 2003). In the study phase, each participant was presented with two consecutive lists of imperatives (e.g., roll the ball) presented at the rate of $8 \mathrm{~s}$ per item. The nouns in the sentences belonged to eight semantic categories, with four items in each. During study of one of the lists, participants were requested to perform the action described by the imperatives, using an object corresponding to the noun (enacted condition). The first measure of recall was a free recall test that followed after each list, and the measure used was the number of sentences (correct verb and noun) recalled in the enacted condition. After the free recall tests described above, participants were given the eight category names into which the nouns could be divided, as cues to remember the nouns. The second measure of recall was the number of nouns (cued) recalled from the enacted condition, and the third measure included was the number of nouns (cued) recalled from nonenacted condition. After an interval of approximately $30 \mathrm{~min}$, filled with other cognitive tests (e.g., the Block Design test described above), the participant was orally and visually presented with the previously studied nouns, intermixed with not previously encountered nouns. The participant indicated whether he or she recognized a noun. The number of hits minus the number of false alarms from the nonenacted condition was used as the fourth measure

Semantic memory functioning. One measure of verbal knowledge and three measures of verbal fluency were used as indicators of semantic memory functioning (cf. Nyberg et al., 2003). The knowledge measure was derived from a 30-item multiple-choice synonym test (Dureman, 1960). Participants selected a synonym to each target word from five alternatives. The total number of correctly selected synonyms in 7 min yielded a measure of verbal knowledge. The first verbal fluency measure was the total number of words with the initial letter $A$ generated in $1 \mathrm{~min}$. The second measure was the total number of professions with the initial letter $B$ generated in $1 \mathrm{~min}$. The third measure represented the total number of five-letter words with the initial letter $M$ generated in $1 \mathrm{~min}$.

\section{Results}

\section{Preliminary Analyses}

Previous cross-sectional (Nyberg et al., 2003; see also Rönnlund et al., 2005) and longitudinal (Lövdén, Rönnlund, et al., 2004) confirmatory factor analyses of the Betula data have provided support for partitioning the declarative memory measures into measures of episodic and semantic memory, based on a similar set of individual measures included here. ${ }^{1}$ In addition, these analyses confirmed both configural and metric measurement invariance across the adult life span (Nyberg et al., 2003) and over time in old age (Lövdén, Rönnlund, et al., 2004). Nevertheless, the empirical background for using the memory measures as indicators of semantic and episodic memory should be confirmed for this sample, time period, and selection of measures. To this end, we specified a standard longitudinal confirmatory factor model (Hertzog \& Schaie, 1986; cf. Lövdén, Rönnlund, et al., 2004) including two factors (episodic memory and semantic memory; see the Method section for the respective indicators) times three occasions. Data for these analyses were provided by the sample of participants who survived and participated at T3 $(n=225)$. This model captured the structure in the data well, $\chi^{2}(207, N=225)=307.03$, comparative fit index $=.967$, root-mean-square error of approximation $=$ .046. Furthermore, forcing longitudinal metric invariance in the estimated factor loadings did not significantly degrade the fit, $\chi^{2}(219, N=225)=317.05$, comparative fit index $=.968$, root-mean-square error of approximation $=.045 ; \Delta \chi^{2}(12, N=$ $225)=10.02, p>.61$. Thus, metric invariance over time was found to be an acceptable hypothesis. The loadings of the indicators on the episodic memory factor were all significant and high (.57-.67 at T1). All loadings on the semantic memory factor were significant and they were high for both the verbal fluency $(.49, .66$, and .74 at T1) and verbal knowledge (.77 at T1) measures. Therefore, on the basis of standard psychometric criteria, the measures included in the present analyses are valid indicators of semantic and episodic memory.

Performance on the Block Design task is often construed as measuring spatial visualization ability but also loads highly on higher-order fluid intelligence constructs such as Cattell's (1971) Gf (e.g., Carroll, 1993). Taken together, the present measures tap important aspects of the basic products of cognition: fluid intelligence (Block Design), crystallized abilities (semantic memory), and episodic memory. The cross-sectional age gradients at T1 support this view: The age correlation was higher for Block Design (-.44) than for a unit-weighted composite of the episodic memory measures (-.36) and a semantic memory composite (-.28). Furthermore, the partial correlation with years of formal education, controlling for age, was higher for semantic memory (.42) than for episodic memory (.27) and Block Design (.35). Thus, the measures showed similar divergent validity with respect to age and sociobiographical associations as those displayed by measures of crystallized and fluid abilities (e.g., Lindenberger \& Baltes, 1997).

By standardizing each individual measure, summing the measures for each construct separately, and standardizing the sums to the T metric $(M=50 ; S D=10)$, we formed three variables (Block Design, episodic memory, and semantic memory) to be included in the cluster analyses. The $\mathrm{T} 1$ sample provided reference values for all occasions. All analyses were performed, and all results are reported in this metric. As shown in Table 2, the 5- and 10-year test-retest correlations are high for all three composites, suggesting lower reliability boundaries of the three variables that are acceptable.

For the cluster analyses, the statistical package SLEIPNER was utilized and the LICUR procedure was followed (Bergman, 1998; Bergman et al., 2003). The first step in this procedure is to identify a residue of outliers separately at each measurement occasion (see Bergman, 1998, for rationale). The participants in the residue are held dissimilar to all other participants. In this study, a participant whose value profile in Block Design, episodic memory, and semantic memory has an averaged squared euclidean distance

${ }^{1}$ As compared with the Lövdén Rönnlund, et al. (2004) and Rönnlund et al. (2005) articles, which reported two-occasion longitudinal data, two indicators (one for each construct) were dropped because they were not measured at $\mathrm{T} 3$. 
Table 2

Correlations Among Block Design, Episodic Memory Performance, and Semantic Memory

Performance

\begin{tabular}{llllllllll}
\hline Measure & 1 & 2 & 3 & 4 & 5 & 6 & 7 & 8 & 9 \\
\hline
\end{tabular}

Time 1

$\begin{array}{llll}\text { 1. Block Design } & - & & \\ \text { 2. Episodic memory } & .41 & \overline{-} & \\ \text { 3. Semantic memory } & .50 & .52 & -\end{array}$

\begin{tabular}{lllllll}
\hline & \multicolumn{7}{c}{ Time 2} \\
4. Block Design & $\mathbf{. 7 5}$ & .37 & .46 & - & & \\
5. Episodic memory & .37 & $\mathbf{. 7 0}$ & .52 & .40 & - & \\
6. Semantic memory & .42 & .50 & $\mathbf{. 8 1}$ & .45 & .53 & - \\
\hline
\end{tabular}

Time 3

\begin{tabular}{llllllllll} 
7. Block Design & $\mathbf{. 7 2}$ & .43 & .39 & $\mathbf{. 7 4}$ & .41 & .42 & - & \\
8. Episodic memory & .31 & $\mathbf{. 6 6}$ & .46 & .37 & $\mathbf{. 7 2}$ & .52 & .47 & - & \\
9. Semantic memory & .44 & .50 & $\mathbf{. 8 0}$ & .46 & .52 & $\mathbf{. 8 0}$ & .46 & .56 & - \\
\hline
\end{tabular}

Note. $n=225$. Test-retest correlations are in bold. All correlations are significant at $p<.001$.

(ASED) of at least 100 to all other participants' value profiles was considered as such an individual. However, neither at T1, T2, or $\mathrm{T} 3$ did the residue analyses locate any outliers. Thus, all available individuals were cluster analyzed.

\section{Typical Profiles of Cognitive Functioning in Old Age}

The second step in LICUR is to perform cluster analyses separately for the data from each measurement occasion $\left(n_{\mathrm{T} 1}=500 ; n_{\mathrm{T} 2}=387\right.$; $\left.n_{\mathrm{T} 3}=225\right)$. The scores in Block Design, episodic memory, and semantic memory constituted the value profile to be analyzed. The method for cluster analysis was Ward's (1963) hierarchical agglomerative method with squared euclidean distances. The guidelines established in LICUR for choosing the number of clusters were applied: (a) A sudden drop in the explained error sum of squares (EESS) of the solution may indicate that a suboptimal number of clusters have been reached, (b) the accepted solution has to be meaningful and the last cluster fusion judged not to obliterate two distinct and theoretically interpretable clusters, (c) the number of clusters should preferably not be more than 15 and cannot normally be expected to be fewer than 5 , and (d) the EESS should preferably exceed $67 \%$. EESS is computed as follows: EESS $=100 \times($ total ESS - ESS of cluster solution $) /$ total ESS, where ESS is the error sum of squares. This measure corresponds to a measure of "explained variation." In the interpretation of the cluster solutions we focus on the cluster centroids; that is, the profile of the means in the three variables for each cluster. At all three time points, the criteria described above indicated that a six-cluster solution was acceptable.

To study stability of the clusters over time, one needs a procedure for comparing the similarity of the different sets of centroids (in our case, the centroids from $\mathrm{T} 1$ and $\mathrm{T} 2$, from $\mathrm{T} 2$ and $\mathrm{T} 3$, and from $\mathrm{T} 1$ and $\mathrm{T} 3$ were compared). The pairwise matching procedure in LICUR first computes the ASED between each pair of centroids, with one centroid coming from the first analysis and the other centroid coming from the second analysis. Then, the two most similar ones become the first match; the two most similar ones among the remaining centroids become the second match and so on until all centroids have been matched. An ASED lower than the average of all within-cluster ASEDs (i.e., the homogeneity coefficient) for a well-functioning cluster solution with homogenous clusters can be regarded as an acceptable match. Bergman et al. (2003) has suggested a limit for the homogeneity coefficient at 100 and, as desirable, 50 (for the $\mathrm{T}$ metric). For considering a match as acceptable, we applied the more stringent level. ${ }^{2}$

The ASEDs between the centroids at the different time points are summarized in Table 3. The ASEDs between matched centroids are in bold. It is evident from Table 3 that structural stability is quite high. In fact, only one marginally acceptable match is found (Cluster A2 with Cluster C2, where $A$ refers to T1 and $C$ refers to T3; ASED $=77$ ). In other words, similar centroids are found at all three time points.

\section{Typical Developmental Paths of Cognitive Functioning in Old Age}

After the centroids had been matched over time, the individuals' cluster membership was cross-tabulated across solutions (i.e., T1 with T2, T2 with T3, and T1 with T3). The experimental dropout, dead, and dementia categories at T2 and T3 were also included in these cross-tabulations. For each cell, we examined whether the cell frequency was significantly higher than expected by chance, indicating a typical developmental path. An exact hypergeometric test was used for this purpose (Bergman \& El-Khouri, 1987). We applied an alpha level at .01 (one-tailed) for the expected cells corresponding to no change in cluster belonging and to transitions

\footnotetext{
${ }^{2}$ To our knowledge, no methods exist for examining the sampling variance of the centroids generated by cluster solutions or for significance testing ASED coefficients between two cluster solutions. Therefore, it is appropriate to address the issues of this aspect of reliability with replication across methods and samples. Such analyses are reported in the Reliability and Validity section.
} 
Table 3

Averaged Squared Euclidean Distances (ASEDs) Between the Centroids from the Three Cluster Solutions (Time 1, Time 2, and Time 3)

\begin{tabular}{|c|c|c|c|c|c|c|c|c|c|c|c|c|c|}
\hline Cluster & B1 & B2 & B3 & B4 & B5 & B6 & $\mathrm{C} 1$ & $\mathrm{C} 2$ & $\mathrm{C} 3$ & $\mathrm{C} 4$ & $\mathrm{C} 5$ & C6 & $\mathrm{HC}$ \\
\hline $\mathrm{A} 1$ & 2 & 128 & 113 & 315 & 253 & 672 & 17 & 274 & 146 & 418 & 214 & 920 & 67 \\
\hline $\mathrm{A} 2$ & 58 & 24 & 14 & 87 & 136 & 359 & 20 & 77 & 25 & 162 & 84 & 545 & 64 \\
\hline A3 & 147 & 121 & 16 & 92 & 54 & 243 & 102 & 125 & 38 & 115 & 29 & 388 & 56 \\
\hline A4 & 310 & 104 & 64 & 3 & 84 & 86 & 214 & 30 & 66 & 7 & 44 & 183 & 71 \\
\hline A5 & 380 & 218 & 131 & 74 & 17 & 62 & 299 & 128 & 169 & 58 & 22 & 139 & 64 \\
\hline A6 & 790 & 429 & 341 & 138 & 212 & 8 & 642 & 222 & 347 & 73 & 199 & 8 & 61 \\
\hline B1 & & & & & & & 10 & 254 & 135 & 407 & 220 & 918 & 78 \\
\hline B2 & & & & & & & 56 & 36 & 61 & 157 & 122 & 531 & 75 \\
\hline B3 & & & & & & & 59 & 77 & 7 & 110 & 52 & 437 & 54 \\
\hline B4 & & & & & & & 205 & 14 & 69 & 12 & 55 & 203 & 65 \\
\hline B5 & & & & & & & 212 & 144 & 146 & 106 & 9 & 254 & 68 \\
\hline B6 & & & & & & & 537 & 171 & 293 & 51 & 136 & 20 & 84 \\
\hline $\mathrm{HC}$ & & & & & & & 99 & 36 & 40 & 48 & 82 & 49 & \\
\hline
\end{tabular}

Note. ASEDs of matched clusters are in bold. A, B, and C indicate the cluster solutions corresponding to Time 1, Time 2, and Time 3, respectively. HC $=$ homogeneity coefficient.

from overall below-mean performance to a dropout category. To guard against the mass significance fallacy, we applied an alpha at .001 (one-tailed) for all other cells.

The main results of this study are shown in Figure 1. The figure displays the centroids for the different clusters sorted from left to right by measurement occasion, and sorted from top to bottom (a) by ascending mean levels at $\mathrm{T} 1$, (b) by the match between $\mathrm{T} 1$ and $\mathrm{T} 2$, and (c) by the match to T2 at T3. It is evident in this figure that similar clusters are found at the three time points. The arrows connecting clusters from different measurement occasions represent typical developmental paths, operationally defined as transition probabilities that are more likely than expected by chance. Demographic characteristics for the different clusters and the dropout categories are summarized in Table 4. From this table, it is apparent that the average chronological age of individuals at a given measurement occasion varies across clusters; that is, certain profiles are more common among older individuals (e.g., Cluster A6), whereas other profiles are more common among younger individuals (e.g., Cluster A1). Nevertheless, to make valid inferences about developmental changes, we need to focus on longitudinal patterns, defined as typical developmental paths over time. These patterns are most clearly discernible in Figure 1.

First, all six T1-T2 cluster pairs and all six T2-T3 cluster pairs show significant individual stability. For example, if an individual starts in Cluster A1 at T1, he or she is 6.1 times (36/5.9) more likely than expected by chance to belong to Cluster B1 (where $B$ refers to T2) at T2. Thus, the general impression is that individuals who do not drop out of the study tend to keep their configuration of the cognitive system over time.

Second, at least three typical developmental paths representing intraindividual change in cognitive profile exist: (a) to start in Cluster A5 and end up in Cluster B6, indicating a major drop in Block Design but otherwise minor changes; (b) to start in Cluster B2 and end up in Cluster $\mathrm{C} 1$, signaling a major increase in Block Design and episodic memory; and (c) to start in Cluster B3 and end in Cluster C5, indicating a major loss in episodic memory but otherwise minor changes. A fourth typical path of individual change is to start in Cluster A2 and end up in Cluster B3, indicating loss in semantic memory but otherwise no large changes. However, Cluster A2 and Cluster B3 are rather similar (ASED = 14), and this path is perhaps best interpreted as indicating stability. Considering the intraindividual change paths in the context of those representing stability suggests that subgroups of individuals from originally cognitively homogenous subgroups develop differently from each other.

Third, individuals in closer proximity of dementia diagnosis $(<5$ years prior to diagnosis) are typically characterized by the lowest levels of functioning in the sample on all parts of the considered cognitive system (i.e., Clusters A6 and B6). In addition, a typical more distal profile preceding dementia diagnosis (5-10 years prior) is to possess relatively low declarative memory functioning (i.e., episodic and semantic memory) but perform around the mean of the sample on Block Design (i.e., Cluster A5 to dementia at T3). It is interesting that it is also typical to start in this dementia-preceding cluster (A5) and end up in Cluster B6 at T2, indicating a loss in Block Design. Together, these patterns may suggest that the developmental path from a relatively differentiated cognitive system, but low declarative memory functioning, toward a profile of lowest functional level across the entire system is a likely typical path of long-term preclinical progression of dementia.

Fourth, Cluster A6, being lowest in the sample throughout the considered cognitive system at $\mathrm{T} 1$, is typically preceding dying between T2 and T3. In other words, individuals in this cluster are typically closer to death than others. It is interesting that the cognitive profile indicated by this cluster does not appear to immediately precede dying (i.e., neither between Cluster A6 at T2 nor between Cluster B6 at T3). However, individuals in this cluster are typically receiving a dementia diagnosis within 5 years, and dementia diagnosis is, not surprisingly, typically followed by death. Thus, the path from Cluster A6 and later death is likely associated with dementia diagnosis.

\section{Replication After Statistically Controlling for Associations to Chronological Age}

As previously noted, some profiles are more common among older individuals, whereas some profiles are more common among 


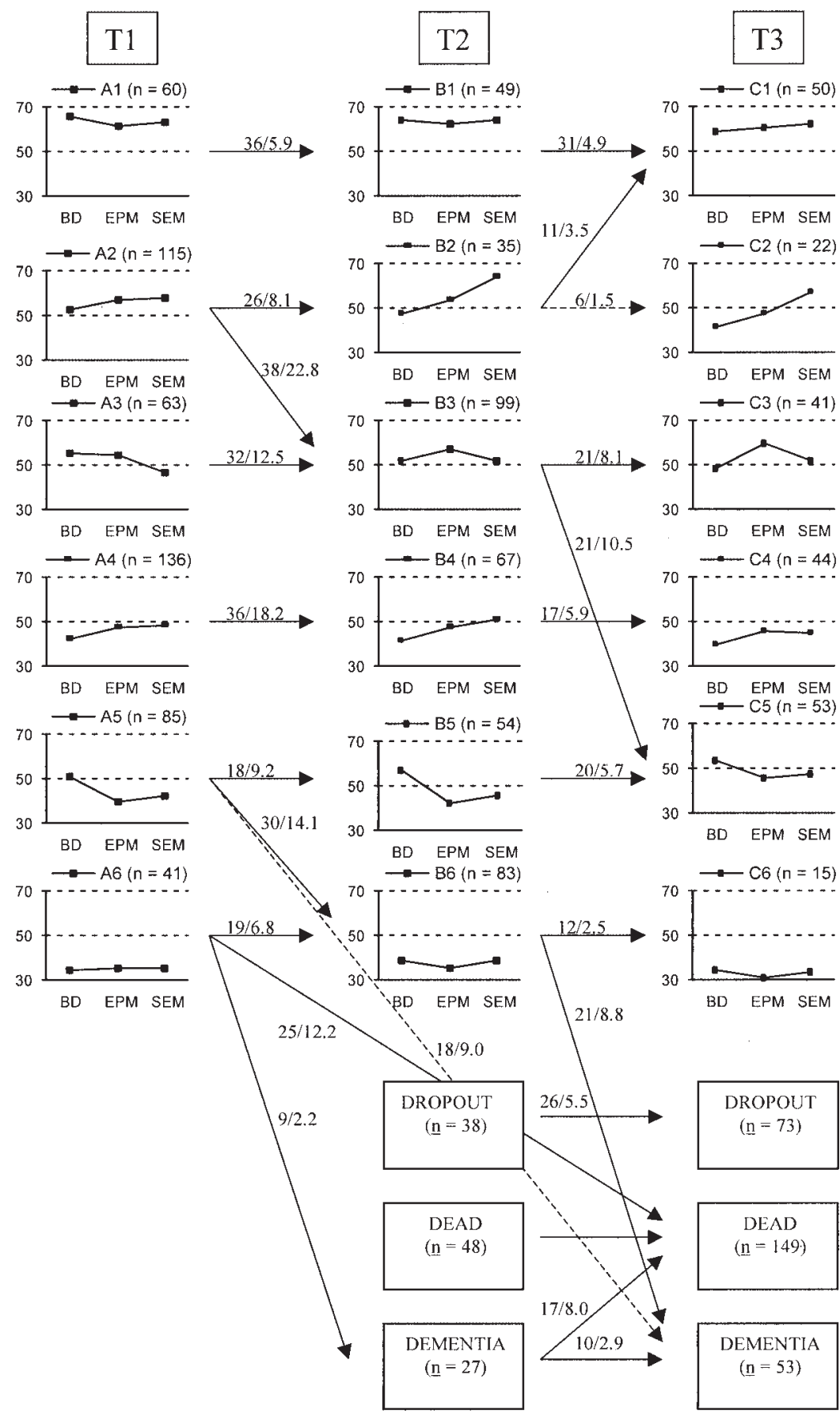

Figure 1. Centroids of the clusters and significant typical developmental paths of cluster membership. Solid arrows indicate significant paths at $p<.001$ (one-tailed), and dashed arrows indicate $p<.01$ (one-tailed). Numerals next to an arrow indicate the observed and expected frequency, respectively. By design, individuals in the dementia group cannot transfer to the dropout group. With this exception only, all naturally possible degrees of freedom are included in the cross-tabulation. Significant T1 to T3 developmental paths are only shown if they apply to the dropout, dementia, or dead groups. In the graphs, the ordinate denotes T-scores $(M=$ $50, S D=10$ ), with $\mathrm{T} 1$ providing reference values. $\mathrm{A}, \mathrm{B}$, and $\mathrm{C}$ indicate the cluster solutions corresponding to Time 1 (T1), Time 2 (T2), and Time 3 (T3), respectively. BD = Block Design; EPM = episodic memory; $\mathrm{SEM}=$ semantic memory 
Table 4

Demographic Characteristics for the Clusters and for the Dropout, Dead, and Dementia Categories

\begin{tabular}{|c|c|c|c|c|c|c|c|c|c|c|c|c|c|c|c|}
\hline \multirow[b]{3}{*}{ Group } & & & & \multicolumn{6}{|c|}{ Chronological age (T1) } & \multicolumn{6}{|c|}{ Years of education (T1) } \\
\hline & \multicolumn{3}{|c|}{$n$} & \multicolumn{2}{|c|}{$\mathrm{T} 1$ (A) } & \multicolumn{2}{|c|}{$\mathrm{T} 2(\mathrm{~B})$} & \multicolumn{2}{|c|}{$\mathrm{T} 3(\mathrm{C})$} & \multicolumn{2}{|c|}{$\mathrm{T} 1$ (A) } & \multicolumn{2}{|c|}{$\mathrm{T} 2(\mathrm{~B})$} & \multicolumn{2}{|c|}{$\mathrm{T} 3(\mathrm{C})$} \\
\hline & $\mathrm{T} 1$ (A) & $\mathrm{T} 2$ (B) & $\mathrm{T} 3(\mathrm{C})$ & $M$ & $S D$ & $M$ & $S D$ & $M$ & $S D$ & $M$ & $S D$ & $M$ & $S D$ & $M$ & $S D$ \\
\hline Cluster 1 & 60 & 49 & 50 & $66.3^{*}$ & 5.8 & $64.6^{*}$ & 5.4 & $63.0 *$ & 4.4 & $11.1^{*}$ & 4.1 & $11.2^{*}$ & 4.0 & $10.3^{*}$ & 3.7 \\
\hline Men & 34 & 25 & 29 & & & & & & & & & & & & \\
\hline Women & 24 & 24 & 21 & & & & & & & & & & & & \\
\hline Cluster 2 & 115 & 35 & 22 & $68.1^{*}$ & 7.1 & 69.0 & 7.8 & 67.5 & 6.9 & $8.9 *$ & 3.4 & 9.1 & 3.0 & 9.3 & 3.1 \\
\hline Men & 52 & 16 & 8 & & & & & & & & & & & & \\
\hline Women & 63 & 19 & 14 & & & & & & & & & & & & \\
\hline Cluster 3 & 63 & 99 & 41 & $66.4^{*}$ & 6.1 & $66.4 *$ & 5.7 & $66.5^{*}$ & 6.0 & 7.6 & 2.4 & 8.2 & 2.7 & 8.6 & 3.7 \\
\hline Men & 27 & 47 & 14 & & & & & & & & & & & & \\
\hline Women & 36 & 52 & 27 & & & & & & & & & & & & \\
\hline Cluster 4 & 136 & 67 & 44 & $72.6^{*}$ & 6.5 & 70.4 & 6.6 & 69.2 & 6.4 & $7.2^{*}$ & 2.3 & 7.8 & 3.4 & 7.1 & 1.7 \\
\hline Men & 58 & 27 & 17 & & & & & & & & & & & & \\
\hline Women & 78 & 40 & 27 & & & & & & & & & & & & \\
\hline Cluster 5 & 85 & 54 & 53 & 71.1 & 6.6 & 69.3 & 7.4 & $65.8^{*}$ & 6.2 & $7.0 *$ & 2.0 & 7.5 & 2.3 & 7.9 & 2.7 \\
\hline Men & 49 & 33 & 28 & & & & & & & & & & & & \\
\hline Women & 36 & 21 & 25 & & & & & & & & & & & & \\
\hline Cluster 6 & 41 & 83 & 15 & $75.2^{*}$ & 6.2 & $74.0 *$ & 5.5 & 73.3 & 4.9 & $6.0 *$ & 1.3 & $6.6^{*}$ & 1.8 & 6.5 & 1.8 \\
\hline Men & 20 & 34 & 6 & & & & & & & & & & & & \\
\hline Women & 21 & 49 & 9 & & & & & & & & & & & & \\
\hline Dropout & & 38 & 73 & & & 70.8 & 6.2 & 70.3 & 6.6 & & & 8.5 & 3.1 & 8.0 & 2.8 \\
\hline Men & & 21 & 30 & & & & & & & & & & & & \\
\hline Women & & 17 & 43 & & & & & & & & & & & & \\
\hline Dead & & 48 & 149 & & & $73.3^{*}$ & 7.2 & $73.9^{*}$ & 6.4 & & & 7.3 & 3.2 & 7.4 & 2.8 \\
\hline Men & & 27 & 86 & & & & & & & & & & & & \\
\hline Women & & 21 & 63 & & & & & & & & & & & & \\
\hline Dement & & 27 & 53 & & & $75.7^{*}$ & 4.9 & $72.8^{*}$ & 5.4 & & & 6.7 & 1.5 & 8.0 & 3.5 \\
\hline Men & & 10 & 22 & & & & & & & & & & & & \\
\hline Women & & 17 & 31 & & & & & & & & & & & & \\
\hline All & 500 & 500 & 500 & 70.0 & 7.1 & 70.0 & 7.1 & 70.0 & 7.1 & 8.0 & 3.1 & 8.0 & 3.1 & 8.0 & 3.1 \\
\hline Men & 240 & 240 & 240 & & & & & & & & & & & & \\
\hline Women & 260 & 260 & 260 & & & & & & & & & & & & \\
\hline
\end{tabular}

Note. A, B, and C indicate the cluster solutions corresponding to Time 1 (T1), Time 2 (T2), and Time 3 (T3), respectively.

$* p<.01$ (for a $z$ test with the total sample treated as the null hypothesis population).

younger individuals. That is, we observed cross-sectional mean age differences among clusters of individuals within each of the three measurement occasions. Because individuals were grouped into homogenous clusters based on their cognitive profile, rather than age, this age heterogeneity of the clusters does not jeopardize the interpretability of typical developmental paths (see the Discussion section). Nevertheless, it is informative to know the extent to which the cluster profiles identified in this study were dependent on the presence of age-associated variance in the cognitive variables. This applies especially to the results involving Cluster A6, because individuals in this cluster are on average older than the mean age of the sample (see Table 4). To address the issue, we performed cluster analysis on data from T1 after statistically removing the association between each cognitive variable and chronological age by regressing the cognitive variables on age and using the residuals in the cluster analysis. The analysis produced clusters similar to the analysis of the original data: An acceptable match between the centroids obtained in the two analyses was found for all six centroids ( $M d n$ ASED $=13$, range $=5-34$ ). Furthermore, the pattern of findings with respect to preclinical dementia profiles was identical to the original analyses. Specifically, if an individual started in Cluster A6 at T1, he or she was 3.2 times $(p<.001)$ more likely than expected by chance to belong to the group of individuals diagnosed for dementia at T2. The pattern obtained with age-residualized data also matched the original finding that Cluster A6 typically precedes impending death between T2 and T3. The high degree of similarity between the original and the age-residualized pattern of results invalidates the objection that the original pattern was induced by cross-sectional age differences.

\section{Reliability and Validity}

It is important to evaluate the quality and trustworthiness of cluster solutions. We addressed this issue in several ways. First, for each cluster, the homogeneity coefficient should be considerably lower than the averaged ASED when all individuals belong to the same cluster (for our T score metric, this distance is 200; owing to scaling by T1 values, it is 225 at T2 and 198 at T3). As a rule of thumb, clusters with coefficients under 100 can be considered as 
acceptably homogeneous (Bergman et al., 2003). The homogeneity coefficients indicated that all clusters were reasonably homogenous $\left(M d n_{\mathrm{T} 1}=64 ; \operatorname{range}_{\mathrm{T} 1}=56-71 ; M d n_{\mathrm{T} 2}=72 ; \operatorname{range}_{\mathrm{T} 2}=\right.$ $54-84 ; M d n_{\mathrm{T} 3}=48 ;$ range $\left._{\mathrm{T} 3}=36-99\right)$.

Second, we computed the EESS for each cluster solution. A value of above $67 \%$ can be regarded as satisfactory (e.g., Bergman et al., 2003). The EESS of the cluster solutions was 67.8 at T1, 69.6 at T2, and 68.1 at T3. In other words, two thirds of the variation is explained by the classifications.

Third, it might be useful to demonstrate that the obtained cluster solutions cannot be regarded as having arisen from an analysis of a similar data set in which all relationships have been removed. Therefore, we performed the same type of cluster analysis that was done on the real data set on 20 random data sets, constructed by random permutation of the columns of the original data set. To consider the solution we originally obtained as warranting a substantive interpretation, we demanded that its EESS should be higher than that of any of the 20 corresponding figures obtained from the random data sets. That is, if this is the case, we reject the null hypothesis that the solution we actually obtained is consistent with a hypothesis of no relationships in the data, at the 5\% level. This procedure for significance testing is principally similar to that of testing a correlation coefficient. The significance tests of the cluster solutions at T1, T2, and T3 all indicated significant structures.

Fourth, in a control analysis with T1 data, a different method of cluster analysis was used (the median method; Hartigan, 1975) to verify that its centroids matched those of the main analysis using Ward's (1963) method. The two sets of centroids matched nicely $(M d n$ ASED $=11 ;$ range $=4-75)$.

Fifth, we compared the T1 centroids derived from the longitudinally studied sample with the centroids obtained from a procedurally identical cluster analysis of an independent validation sample, tested for the first time at T2 $(n=500 ; M d n$ age $=70$; age range $=60-80)$. The measures for the validation sample were standardized to the $\mathrm{T}$ metric according to the means and standard deviations from the longitudinal sample at T1. An acceptable match between the centroids obtained in the two samples was found at least for five of the centroids ( $M d n$ ASED $=6$, range $=$ 2-29). The centroid not replicated (A5) had an ASED of 120 to its counterpart in the validation sample.

\section{Discussion}

Two major findings result from the present person-oriented analysis of longitudinal cognitive changes in old age. First, most individuals not dropping out from the study tended to keep their cognitive profile over time. Second, a minority of individuals followed typical (i.e., nonrandom) developmental paths of intraindividual change in cognitive profiles. Together, these two findings confirm our central hypothesis that subgroups of individuals develop differently from each other, but the results are also consistent with earlier univariate results demonstrating relatively high time-based stability of interindividual differences in intraindividual change in old age (e.g., Hultsch et al., 1998; Lövdén, Rönnlund, et al., 2004). It is interesting that proponents of general and process-specific views of cognitive aging both have tended to tacitly accept the assumption that cognitive aging is uniform in the sense that structural relations among tasks and underlying cognitive processes are invariant across individuals. The present findings question this assumption by demonstrating interindividual differences in intraindividual multivariate patterns of change. Some or even most individuals might undergo senescent changes in intellectual performance in a relatively global manner, but others may experience selective changes in a subset of cognitive functions. This is not to be understood as if the results suggest that there are no levels of analysis where a uniformity-of-nature assumption holds, or as if general mechanisms of development are not of primary interest. However, to better understand cognitive aging, it may be necessary to investigate its antecedents at the level of the individual and to allow for interindividual variability in the importance of different biological and sociocultural mechanisms (cf. Lövdén \& Lindenberger, 2005).

Several results concerning the developmental progression of dementia were obtained. A typical early preclinical dementia profile was characterized by relatively low episodic and semantic memory but mean level performance on Block Design. This result is consistent with previous studies highlighting episodic memory deficits in the preclinical stages of dementia (e.g., Bäckman et al., 2001; Small et al., 1997) and with studies suggesting that deficits in cognitive functions that are relatively intact in old age (in this study, semantic memory) may distinguish dementia from aging (Branconnier et al., 1982; Small et al., 1997; see also Spaan et al., 2003). Individuals in the later preclinical stages, however, are typically characterized by the lowest levels of functioning in the sample throughout the considered cognitive system (see also Bäckman et al., 2002; Fabrigoule et al., 1998). Together with the typical developmental path from the early to the late preclinical profile (i.e., Cluster A5 to Cluster B6), the results describe the preclinical phase of dementia as a development from relatively low declarative memory functioning toward a profile of globally low functional levels. These results illustrate the importance of considering that the nature of the preclinical profile may predictably change as a function of proximity to today's diagnosis criteria. For early and reliable diagnosis of dementia, such dynamic information may add important information to static information.

No profile appeared to directly precede death. However, being low across the considered cognitive system (i.e., belonging to Cluster A6) was typically associated with dying within the 10-year frame of this study; that is, individuals in this cluster were typically closer to death than the others. It is interesting that it was also typical to start out in this cluster and become demented within 5 years, and, not surprisingly, dementia was typically followed by death. Taken this pattern together with the description of the preclinical dementia phase, the results describe a developmental cascade of low declarative memory, low functioning across the considered cognitive system, dementia diagnosis, and finally death. In a sense, this path of cognitive development is mortality related, but the results suggest that dementia to some extent hide behind this relation in this sample, or alternatively, that individuals in a terminal decline phase often might be diagnosed for dementia.

It is always important to evaluate the trustworthiness of empirical findings, but because the methods applied here are nonstandard in cognitive aging research, we devoted particular effort to this task. The results indicated that the clusters were reasonably homogenous, that the cluster solutions cannot be regarded as arisen 
from an analysis of random data, that a different method of cluster analysis yielded similar centroids, and that five out of six centroids were externally replicated. In addition, the fact that the centroids replicate across time adds credibility to the quality of the solutions. On the basis of these methodological efforts, the reliability and validity of the results can be judged satisfactory.

A major reason for choosing the LICUR procedure for our individual-oriented approach was its robustness and suitability for the analysis of long-term longitudinal panel data. We also think that the procedure incorporates some of the central messages from the individual-oriented approach: It naturally reflects the dynamic, multivariate, and interactionistic nature of individuals' cognitive development; it clearly incorporates a direct methodological attempt to structure unknown heterogeneity; and it separates and relates intraindividual and interindividual differences. Accordingly, the procedure fosters a data-analytic perspective that matches the individual-oriented theoretical agenda more closely than most other data-analytic approaches to long-term longitudinal panel data such as longitudinal factor analysis. The procedure also covers a wider range of applications than the one presented here. For example, it can test whether certain developmental transitions occur less rather than more frequently than expected by change (e.g., antitypical transitions; von Eye, 1990; see also Bergman et al., 2003). To illustrate, the processing speed theory (Salthouse, 1996) would predict that potential developmental paths representing decrements in perceptual speed accompanied by stability or increase in other cognitive abilities should be less frequently observed than expected by chance. Furthermore, the procedure naturally focuses on sequential multivariate changes, such as the preclinical developmental cascade reported here, that are more difficult to identify with traditional longitudinal methods.

At the same time, cluster-analytic procedures such as LICUR are not without limitations, and it might be appropriate to dwell a while on them. Cluster analyses are often criticized for ambiguity in choosing the number of clusters. Though the LICUR stopping rules have proved useful to overcome this problem (e.g., Bergman, 1998; Bergman et al., 2003), the clusters must not be interpreted to represent the only possible or existing types of cognitive profiles in old age. However, if hierarchical cluster analysis is used, as was the case here, there is a strong correspondence between the cluster solution with $c$ clusters and with $c+1$ clusters, with the $c$ cluster solution resulting from the fusion of two clusters in the $c+$ 1 solution and with all the other clusters being identical. Indeed, stepping up or down one level in hierarchy of clusters does not change any of the substantive results reported here. In this sense, the issue is less critical than the corresponding problem for standard factor analysis, where the number of factors chosen often provides the fundamental empirical basis for theoretical conclusions.

In light of our individual-oriented approach, the interpretation of the cluster centroids should be clarified: Because the values on the variables are standardized with the sample as reference, they cannot be interpreted in an absolute intraindividual sense. To illustrate, the centroid of low declarative memory performance but normal Block Design performance (Cluster A5) cannot be interpreted as if these individuals' fluid abilities are better functioning than their declarative memory in the absolute sense. In other words, because behavioral sciences generally lack the tools of absolute scales, each value has to be interpreted with reference to the sample (see Bergman et al., 2003, for an overview). A related scaling problem could be principally applied to the interpretation of developmental paths: Most of the individual changes in cognitive profile constitute ordinal interactions. As such, these changes cannot, in the strict sense, be unambiguously interpreted (e.g., Dunn \& Kirsner, 1988; Loftus, 1978). Of course, this scaling problem has plagued behavioral sciences for a long time and is by no means specific to this study.

Out of commonly acknowledged validity threats to longitudinal studies, retest effects have probably received least attention (but see Lövdén, Ghisletta, \& Lindenberger, 2004; Rabbitt, Diggle, Smith, Holland, \& McInnes, 2001; Rönnlund et al., 2005; Schaie, 1988, 1996). From previous research with the cognitive battery in Betula, we know that 5-year retest effects are present at least for episodic memory but not for our semantic memory variable (Rönnlund et al., 2005). Overall, the effect is small (0.15 SD). Though we cannot rule out that retest effects have influenced the present results, these effects are unlikely to have affected the observed cluster solutions and cluster transitions in a substantial manner.

Note also that, owing to abundant missing values stemming from difficulties in testing individuals with dementia, we were not able to include these individuals in the cluster analyses. This means that the measurement model, rather that empirically exploring it, a priori assumes that these individuals belong to a homogeneous group different from individuals included in the analysis $^{3}$ - and that the cognitive pattern and developmental path after and immediately before diagnosis is unknown. These aspects obviously further underscore that it is worthwhile to invest considerable resources into minimizing missing data.

Finally, we note that each cluster analysis at each measurement occasion was performed on an age-heterogeneous sample. Certainly, this feature leads to a confounding of chronological age with the profiles within each cluster analyses. For example, the individuals in the clusters characterized by globally low performance (Clusters A6, B6, and C6) were on average older than the mean age of the sample. Considering that the prevalence of dementia increases dramatically with age in this age range, one might argue that the reason why individuals in these clusters typically developed dementia is the age of the individuals in the clusters. However, the analyses on data statistically controlled for the association to chronological age recovered similar centroids at $\mathrm{T} 1$ and produced identical patterns of results with respect to dementia. Thus, the present results are not artifacts of cross-sectional agerelated variance. Furthermore, chronological age does not, obviously, cause dementia and does not, in itself, contain any explanatory capacity. Surely, the state of the cognitive system must be closer to the causal structures behind dementia than time passed since birth. In other words, when it comes to studying cognitive aging, we generally prefer to give interpretational priority to individuals' patterns of cognitive functioning over chronological age. In doing so we echo classic calls for replacing chronological age with variables closer to the developmental process of interest (Wohlwill, 1970; see also Lövdén \& Lindenberger, 2005; S.-C. Li

\footnotetext{
${ }^{3}$ We thank P. B. Baltes for drawing our attention to this point.
} 
\& Schmiedek, 2002; Sliwinski, Hofer, Hall, Buschke, \& Lipton, 2003). The spirit of dynamic systems theories (e.g., Ford \& Lerner, 1992; Gottlieb, 1992; Wohlwill, 1973) inherent in the LICUR procedure, reflected by allowing the cross-sectional cognitive profiles to determine both future cognitive states and developmental paths, offers a viable methodological basis for such endeavors.

To conclude, the individual-oriented approach advocates a focus on the individual that captures the multivariate and dynamic nature of individual development, clearly separates intraindividual and interindividual differences, and structures heterogeneity. The demonstrated methodological approach is not necessarily the best one in terms of implementing these messages. Combinations of variable-oriented and pattern-oriented approaches, although often of univariate nature, are promising approaches to the direct structuring of developmental change, and these methods may sometimes be preferred over the "snapshot and linking" procedure applied here. The multivariate, single subject, repeated measures design (Jones \& Nesselroade, 1990) is a necessary tool for better understanding intellectual aging. In a similar vein, measurement bursts might be necessary for providing empirical information that captures the dynamic properties of behavior and for separating more enduring change from short-term fluctuation in behavior (Nesselroade \& Salthouse, 2004). However, the LICUR procedure is a relatively robust and flexible approach to analyzing longitudinal panel data and deserves a place among the individualoriented tools in the basic tool chest. In this study, the procedure demonstrated systematic interindividual differences in multivariate patterns of change. Furthermore, the results described the progression of dementia as a developmental cascade of low declarative memory, low functioning across the considered system, dementia diagnosis, and, finally, death.

\section{References}

Allen, P. A., Hall, R. J., Druley, J. A., Smith, A. F., Sanders, R. E., \& Murphy, M. D. (2001). How shared are age-related influences on cognitive and noncognitive variables? Psychology and Aging, 16, 532-549.

Allport, G. W. (1937). Personality: A psychological interpretation. New York: Holt, Rinehart, \& Winston.

Almqvist, O., Fratiglioni, L., Agüero-Torres, H., Viitanen, M., \& Bäckman, L. (1999). Cognitive support at episodic encoding and retrieval: Similar patterns of utilization in community-based samples of Alzheimer's disease and vascular dementia patients. Journal of Clinical and Experimental Neuropsychology, 21, 816-830.

American Psychiatric Association. (2000). Diagnostic and statistical manual of mental disorders (4th ed., text rev.). Washington, DC: Author.

Anstey, K. J., \& Smith, G. A. (1999). Interrelationships among biological markers of aging, health, activity, acculturation, and cognitive performance in late adulthood. Psychology and Aging, 14, 605-618.

Bäckman, L., Laukka, E. J., Wahlin, A., Small, B. J., \& Fratiglioni, L. (2002). Influences of preclinical dementia and impending death on the magnitude of age-related cognitive deficits. Psychology and Aging, 17, 435-442.

Bäckman, L., Small, B. J., \& Fratiglioni, L. (2001). Stability of the preclinical episodic memory deficit in Alzheimer's disease. Brain, 124, 96-102.

Baltes, P. B., \& Lindenberger, U. (1997). Emergency of a powerful connection between sensory and cognitive functions across the adult life span: A new window to the study of cognitive aging? Psychology and Aging, 12, 12-21.
Baltes, P. B., \& Nesselroade, J. R. (1979). History and rationale of longitudinal research. In J. R. Nesselroade \& P. B. Baltes (Eds.), Longitudinal research in the study of behavior and development (pp. 1-39). New York: Academic Press.

Baltes, P. B., Reese, H. W., \& Nesselroade, J. R. (1977). Life-span development psychology: Introduction to research methods. Monterey, CA: Brooks/Cole.

Berg, S. (1996). Aging, behavior, and terminal decline. In J. E. Birren \& K. W. Schaie (Eds.), Handbook of the psychology of aging (4th ed., pp. 323-337). New York: Academic Press.

Bergman, L. R. (1998). A pattern-oriented approach to studying individual development: Snapshots and processes. In R. B. Cairns, L. R. Bergman, \& J. Kagan (Eds.), Methods and models for studying the individual (pp. 83-121). Thousand Oaks, CA: Sage.

Bergman, L. R., \& El-Khouri, B. M. (1987). EXACON: A Fortran 77 program for the exact analysis of single cells in a contingency table. Educational and Psychological Measurement, 47, 155-161.

Bergman, L. R., \& Magnusson, D. (1997). A person-oriented approach in research on developmental psychopathology. Development \& Psychopathology, 9, 291-319.

Bergman, L. R., Magnusson, D., \& El-Khouri, B. M. (2003). Studying individual development in an interindividual context: A person-oriented approach. Mahwah, NJ: Erlbaum.

Block, J. (1971). Lives through time. Berkeley, CA: Bancroft Books.

Borsboom, D., Mellenbergh, G. J., \& van Heerden, J. (2003). The theoretical status of latent variables. Psychological Review, 110, 203-219.

Bosworth, H. B., \& Schaie, K. W. (1999). Survival effects in cognitive function, cognitive style, and sociodemographic variables in the Seattle Longitudinal Study. Experimental Aging Research, 25, 121-139.

Branconnier, R. J., Cole, J. O., Spera, K. F., \& DeVitt, D. R. (1982). Recall and recognition as diagnostic indices of malignant memory loss in senile dementia: A Bayesian analysis. Experimental Aging Research, 8, 189193.

Carroll, J. B. (1993). Human cognitive abilities: A survey of factor analytic studies. Cambridge, England: Cambridge University Press.

Cattell, R. B. (1971). Abilities: Their structure, growth, and action. Boston: Houghton Mifflin.

Collins, L. M., \& Sayer, A. (Eds.). (2001). New methods for the analysis of change. Washington, DC: American Psychological Association.

Collins, L. M., \& Wugalter, S. E. (1992). Latent class models for stagesequential synamic latent variables. Multivariate Behavioral Research, $27,131-157$.

Dunn, J. C., \& Kirsner, K. (1988). Discovering functionally independent mental processes: The principle of reversed association. Psychological Review, 95, 91-101.

Dureman, I. (1960). SRB:1. Stockholm, Sweden: Psykologiförlaget.

Elias, M. F., Beiser, A., Wolf, P. A., Au, R., White, R. F., \& D'Agostino, R. B. (2000). The preclinical phase of Alzheimer's disease: A 22-year prospective study of the Framingham cohort. Archives of Neurology, 57, $808-813$.

Fabrigoule, C., Rouch, I., Taberly, A., Letenneur, L., Commenges, D., Mazaux, J.-M., et al. (1998). Cognitive process in preclinical phase of dementia. Brain, 121, 135-141.

Folstein, M. G., Folstein, S. E., \& McHugh, P. R. (1975). "Mini-mental state": A practical method for grading the cognitive state of patients for the clinician. Journal of Psychiatric Research, 12, 189-198.

Ford, D. H., \& Lerner, R. M. (1992). Developmental systems theory: An integrative approach. Newbury Park, CA: Sage.

Gottlieb, G. (1992). Individual development and evolution: The genesis of novel behavior. New York: Oxford University Press.

Hartigan, J. A. (1975). Clustering algorithms. New York: Wiley.

Hertzog, C. (1985). An individual differences perspective: Implications for cognitive research in gerontology. Research on Aging, 7, 7-45. 
Hertzog, C., \& Schaie, K. W. (1986). Stability and change in adult intelligence: I. Analysis of longitudinal covariance structures. Psychology and Aging, 1, 159-171.

Hodges, J. R., \& Patterson, K. P. (1995). Is semantic memory consistently impaired early in the course of Alzheimer's disease? Neuroanatomical and diagnostic implications. Neuropsychologia, 33, 441-459.

Hofer, S. M., Berg, S., \& Era, P. (2003). Evaluating the interdependence of aging-related changes in visual and auditory acuity, balance, and cognitive functioning. Psychology and Aging, 18, 285-305.

Hofer, S. M., \& Sliwinski, M. J. (2001). Understanding aging: An evaluation of research designs for assessing the interdependence of ageingrelated changes. Gerontology, 47, 341-352.

Hultsch, D. F., Hertzog, C., Dixon, R. A., \& Small, B. J. (1998). Memory change in the aged. New York: Cambridge University Press

Jacobs, D. M., Sano, M., Dooneief, G., Marder, K., Bell, K. L., \& Stern, Y. (1995). Neuropsychological detection and characterization of preclinical Alzheimer's disease. Neurology, 45, 957-962.

Jones, C. J., \& Nesselroade, J. R. (1990). Multivariate, replicated, singlesubject, repeated measures design and P-technique factor analysis: A review of intraindividual change studies. Experimental Aging Research, 16, 171-183.

Kalaria, R. N., \& Ballard, C. (1999). Overlap between pathology of Alzheimer's disease and vascular dementia. Alzheimer's Disease and Associated Disorders, 13, 115-123.

Kleemeier, R. W. (1962). Intellectual changes in the senium. Proceedings of the American Statistical Association, 1, 290-295.

Li, K. Z. H., \& Lindenberger, U. (2002). Relations between aging sensory/ sensorimotor and cognitive functions. Neuroscience \& Biobehavioral Reviews, 26, 777-783.

Li, S.-C. (2003). Biocultural orchestration of developmental plasticity across levels: The interplay of biology and culture in shaping the mind and behavior across the life span. Psychological Bulletin, 129, 171-194.

Li, S.-C., \& Schmiedek, F. (2002). Age is not necessarily aging: Another step toward understanding the "clocks" that time aging. Gerontology, 48, $5-12$.

Lindenberger, U., \& Baltes, P. B. (1994). Sensory functioning and intelligence in old age: A strong connection. Psychology and Aging, 9, 339-355.

Lindenberger, U., \& Baltes, P. B. (1997). Intellectual functioning in old and very old age: Cross-sectional results from the Berlin Aging Study. Psychology and Aging, 12, 410-432.

Lindenberger, U., \& Pötter, U. (1998). The complex nature of unique and shared effects in hierarchical linear regression: Implications for developmental psychology. Psychological Methods, 3, 218-230.

Little, T. D., Schnabel, K. U., \& Baumert, J. (Eds.). (2000). Modeling longitudinal and multilevel data (pp. 187-200). Mahwah, NJ: Erlbaum.

Loftus, G. R. (1978). On interpretation of interactions. Memory \& Cognition, 6, 312-319.

Lövdén, M., Ghisletta, P., \& Lindenberger, U. (2004). Cognition in the Berlin Aging Study (BASE): The first ten years. Aging, Neuropsychology, and Cognition, 11, 104-133.

Lövdén, M., \& Lindenberger, U. (2005). Development of intellectual abilities in old age: From age gradients to individuals. In O. Wilhelm \& R. W. Engle (Eds.), Understanding and measuring intelligence (pp. 203-222). Thousand Oaks, CA: Sage.

Lövdén, M., Rönnlund, M., Wahlin, Å., Bäckman, L., Nyberg, L., \& Nilsson, L.-G. (2004). The extent of stability and change in episodic and semantic memory in old age: Demographic predictors of level and change. Journals of Gerontology, Series B: Psychological Sciences and Social Sciences, 59, P130-P134.

Luszcz, M. A., \& Bryan, J. (1999). Toward understanding age-related memory loss in late adulthood. Gerontology, 45, 2-9.

Magnusson, D. (2001). The holistic-interactionistic paradigm: Some direc- tions for empirical developmental research. European Psychologist, 6, $153-162$.

Molenaar, P. C. M., Huizenga, H. N., \& Nesselroade, J. R. (2003). The relationship between the structure of interindividual and intraindividual variability. A theoretical and empirical vindication of developmental systems theory. In U. M. Staudinger \& U. Lindenberger (Eds.), Understanding human development: Dialogues with lifespan psychology (pp. 339-360). Dordrecht, Netherlands: Kluwer Academic.

Muthén, B., \& Muthén, L. K. (2000). Integrating person-centered and variable-centered analyses: Growth mixture modeling with latent trajectory classes. Alcoholism: Clinical and Experimental Research, 24, 882891.

Nagin, D. S., \& Tremblay, R. E. (2001). Analyzing developmental trajectories of distinct but related behaviors: A group-based method. Psychological Methods, 6, 18-34.

Nesselroade, J. R., \& Ford, D. H. (1985). P-technique comes of age: Multivariate, replicated, single-subject designs for research on older adults. Research on Aging, 7, 46-80.

Nesselroade, J. R., \& Ghisletta, P. (2000). Beyond statistic concepts in modeling behavior. In L. R. Bergman, R. B. Cairns, L.-G. Nilsson, \& L. Nystedt (Eds.), Developmental science and the holistic approach (pp. 121-135). Mahwah, NJ: Erlbaum.

Nesselroade, J. R., \& Salthouse, T. A. (2004). Methodological and theoretical implication of intraindividual variability in perceptual motor performance. Journals of Gerontology, Series B: Psychological Sciences and Social Sciences, 59, 49-55.

Nilsson, L.-G., Adolfsson, R., Bäckman, L., de Frias, C. M., Molander, B., \& Nyberg, L. (2004). Betula: A prospective cohort study on memory, health, and aging. Aging, Neuropsychology, and Cognition, 11, 134148 .

Nilsson, L.-G., Bäckman, L., Erngrund, K., Nyberg, L., Adolfsson, R., Bucht, G., et al. (1997). The Betula prospective cohort study: Memory, health, and aging. Aging, Neuropsychology, and Cognition, 4, 1-32.

Nyberg, L., Maitland, S. B., Rönnlund, M., Bäckman, L., Dixon, R. A., Wahlin, A., \& Nilsson, L.-G. (2003). Selective adult age differences in an age-invariant multifactor model of declarative memory. Psychology and Aging, 18, 149-160.

Rabbitt, P. M. A. (1993). Does it all go together when it goes? The nineteenth Bartlett memorial lecture. Quarterly Journal of Experimental Psychology: Human Experimental Psychology, 46(A), 385-434.

Rabbitt, P., Diggle, P., Smith, D., Holland, F., \& McInnes, L. (2001). Identifying and separating the effects of practice and of cognitive ageing during a large longitudinal study of elderly community residents. $\mathrm{Neu}$ ropsychologia, 39, 532-543.

Riegel, K. F., \& Riegel, R. M. (1972). Development, drop, and death. Developmental Psychology, 6, 306-319.

Rönnlund, M., Nyberg, L., Bäckman, L., \& Nilsson, L.-G. (2005). Stability, growth, and decline in adult life span development of declarative memory: Cross-sectional and longitudinal data from a population-based study. Psychology and Aging, 20, 3-18.

Salthouse, T. A. (1996). The processing speed theory of adult age differences in cognition. Psychological Review, 103, 403-428.

Salthouse, T. A., \& Czaja, S. J. (2000). Structural constraints on process explanations in cognitive aging. Psychology and Aging, 15, 44-55.

Schaie, K. W. (1988). Internal validity threats in studies of adult cognitive development. In M. L. Howe \& C. J. Brainard (Eds.), Cognitive development in adulthood: Progress in cognitive development research (pp. 241-272). New York: Springer.

Schaie, K. W. (1989). Individual differences in rate of cognitive change in adulthood: In V. L. Bengtson \& K. W. Schaie (Eds.), The course of later life: Research and reflections (pp. 68-83). New York: Springer

Schaie, K. W. (1996). Intellectual development in adulthood: The Seattle longitudinal study. New York: Cambridge University Press. 
Schmiedek, F., \& Li, S.-C. (2004). Towards an alternative representation for disentangling age-associated differences in general and specific cognitive abilities. Psychology and Aging, 19, 40-56.

Singer, T., Verhaeghen, P., Ghisletta, P., Lindenberger, U., \& Baltes, P. B. (2003). The fate of cognition in very old age: Six-year longitudinal findings in the Berlin Aging Study (BASE). Psychology and Aging, 18, $318-331$.

Sliwinski, M. J., Hofer, S. M., \& Hall, C. (2003). Correlated and coupled cognitive change in older adults with and without preclinical dementia. Psychology and Aging, 18, 672-683.

Sliwinski, M. J., Hofer, S. M., Hall, C., Buschke, H., \& Lipton, R. B. (2003). Modeling memory decline in older adults: The importance of preclinical dementia, attrition, and chronological age. Psychology and Aging, 18, 658-671.

Small, B. J., \& Bäckman, L. (1999). Time to death and cognitive performance. Current Directions in Psychological Science, 8, 168-172.

Small, B. J., Fratiglioni, L., von Strauss, E., \& Bäckman, L. (2003). Terminal decline and cognitive performance in very old age: Does cause of death matter? Psychology and Aging, 18, 193-202.

Small, B. J., Herlitz, A., Fratiglioni, L., Almqvist, O., \& Bäckman, L. (1997). Cognitive predictors of incident Alzheimer's disease: A prospective longitudinal study. Neuropsychology, 11, 413-420.

Smith, L. B., \& Thelen, E. (2003). Development as a dynamic system. Trends in Cognitive Sciences, 7, 343-348.

Spaan, P. E. J., Raaijmakers, J. G. W., \& Jonker, C. (2003). Alzheimer's disease versus normal ageing: A review of the efficiency of clinical and experimental memory measures. Journal of Clinical and Experimental Neuropsychology, 25, 216-233.

Valsiner, J. (1984). Two alternative epistemological frameworks in psychology: The typological and variational modes of thinking. The Journal of Mind and Behavior, 5, 449-470.

Verhaeghen, P., \& Salthouse, T. A. (1997). Meta-analyses of agecognition relations in adulthood: Estimates of linear and nonlinear age effects and structural models. Psychological Bulletin, 122, 231-249.

von Eye, A. (1990). Introduction to configural frequency analysis: The search for types and antitypes in cross-classification. Cambridge, England: Cambridge University Press.

Ward, J. H. (1963). Hierarchical grouping to optimize an objective function. Journal of the American Statistical Association, 58, 236-244.

Wechsler, D. (1981). Wechsler Adult Intelligence Scale—Revised: Manual. New York: Psychological Corporation.

Wilson, R. S., Beckett, L. A., Barnes, L. L., Schneider, J. A., Bach, J., Evans, D. A., \& Bennett, D. A. (2002). Individual differences in rates of change in cognitive abilities of older persons. Psychology and Aging, 17 , 179-193.

Wohlwill, J. F. (1970). The age variable in psychological research. Psychological Review, 77, 49-64.

Wohlwill, J. F. (1973). The study of behavioral development. New York: Academic Press.

Received January 19, 2004

Revision received January 4, 2005

Accepted February 22, 2005

\section{Low Publication Prices for APA Members and Affiliates}

Keeping you up-to-date. All APA Fellows, Members, Associates, and Student Affiliates receive-as part of their annual dues-subscriptions to the American Psychologist and APA Monitor. High School Teacher and International Affiliates receive subscriptions to the APA Monitor, and they may subscribe to the American Psychologist at a significantly reduced rate. In addition, all Members and Student Affiliates are eligible for savings of up to $60 \%$ (plus a journal credit) on all other APA journals, as well as significant discounts on subscriptions from cooperating societies and publishers (e.g., the American Association for Counseling and Development, Academic Press, and Human Sciences Press).

Essential resources. APA members and affiliates receive special rates for purchases of APA books, including the Publication Manual of the American Psychological Association, and on dozens of new topical books each year.

Other benefits of membership. Membership in APA also provides eligibility for competitive insurance plans, continuing education programs, reduced APA convention fees, and specialty divisions.

More information. Write to American Psychological Association, Membership Services, 750 First Street, NE, Washington, DC 20002-4242. 